\title{
A NONLINEAR ELLIPTIC PROBLEM RELATED TO FLOWING GRANULAR MATERIALS
}

\author{
PIERRE A. GREMAUD*
}

\begin{abstract}
Similarity solutions for the flow of granular materials are constructed. Unlike previous work, the present approach can be applied to non-axisymmetric containers. The steady state equations are reduced to a nonlinear Helmholtz on a subdomain of the sphere. Existence and local uniqueness of the solutions are established. A spectral numerical method using Fourier/ChebyshevGauss-Radau collocation for discretization and Newton-GMRES as solver is proposed and implemented. Corresponding numerical experiments are discussed.
\end{abstract}

Key words. elliptic, granular, similarity, spectral

AMS subject classifications. $65 \mathrm{~N} 35,35 \mathrm{~J} 65,76 \mathrm{~T} 25$

1. Introduction. This paper is about the determination of the flow of granular material under gravity in hoppers of simple geometry. This problem is common to many industrial processes. In spite of its apparent simplicity, it presents a formidable array of difficulties. In practice, the withdrawal of stored materials from hoppers and bins is well known to be problematic. No flow, segregation, flooding (uncontrolled flow) and structural failures are often encountered [11]. Improved design criteria are sought through a better understanding of such flows. All the previous contributions we are aware of [4], [5], [7]-[9], [13]-[17], [20], to cite but a few, deal only with axisymmetric containers. This significant restriction is removed here.

Apart from the geometry of the hopper, two important factors are the internal friction of the material and the friction between wall and material. Those parameters are further discussed in Section 2. The flows themselves can reach from established mass flows where the material moves and deforms everywhere in a "smooth way" to highly time-dependent funnel flows where motion only takes place in the central part of the silo [14]. In the first case, steady state models are often appropriate. Indeed, current design criteria are based on mass flow calculations through the resolution of steady state models, a view point that is also taken in this paper. Throughout, the material is assumed to be an incompressible, perfectly plastic, cohesionless Coulomb powder with a yield surface of von Mises type. Further, the eigenvectors of the strain rate and stress tensors are assumed to be parallel. Those assumptions are standard in this field, although the alignment condition is somewhat controversial. Those issues are commented on in Section 2. Apart from the above constitutive relations, the equations derive from the basic principles of Continuum Mechanics: conservation of mass and momentum. Conservation of energy does not enter the problem as heat losses through friction influence the temperature which is not related in any significant way to the variables under study.

A study of some of the mathematical properties of the model corresponding to the above assumptions can be found in [17]. The steady state equations can be elliptic, hyperbolic or have no definite type depending in the material parameters.

Much remains to be done about the analysis of the above problems and as a result, the design of mass flow hoppers still greatly relies on an observation due to

\footnotetext{
* Department of Mathematics and Center for Research in Scientific Computation, North Carolina State University, Raleigh, NC 27695-8205, USA (gremaud@math.ncsu.edu). Partially supported by the Army Research Office (ARO) through grant DAAD19-99-1-0188 and by the National Science Foundation (NSF) through grant DMS-9818900.
} 
Jenike [8], [9] in the late 1950's. It was noticed that for three dimensional conical hoppers as well as for two-dimensional wedge shaped hoppers the above equations admit similarity solutions. Those radial solutions correspond to particle paths that are radial lines. The similarity is reflected in scalings of the stresses and velocities with respect to the radial distance $r$ from the vertex of the hopper. In those simple geometries, the equations for the radial fields reduce to systems of ODEs that can be easily numerically integrated. The stability of the solutions to perturbations, change of the geometry (opening angle) and/or physical properties of the hoppers were studied in [4], [15] and [16] through numerical resolutions of the full PDE system (in [16], the material is assumed to be compressible) and in [5] and [15] through linear stability studies. Experimental evidences [14] confirm the important role played by the radial fields in practice.

The purpose of this paper is to show that Jenike's construction of similarity solutions can be generalized to general mathematical cones, i.e., pyramidal domains of arbitrary cross section. This is significant as previous works in this area deal exclusively with axisymmetric containers, even though those are the exception rather than the rule in practice (see e.g. [18] for remarks on the influence of the hopper geometry on the flowing properties). The loss of axisymmetry considerably complicates the structure of the stress tensor and the ensuing equations. As is the case for Jenike's radial solutions, the radial structure leads to significant simplifications. However, instead of ODEs, the radial stress field must here be obtained through the resolution of a nonlinear Helmholtz equation in a subdomain of the sphere.

The paper is organized as follows. The model, geometry and physical assumptions are discussed in Section 2. An existence and local uniqueness result is stated and proved in Section 3. In Section 4, a pseudospectral numerical method is proposed. It uses Fourier collocation in longitude and Chebyshev-Gauss-Radau collocation in latitude, to account for the boundary conditions at hand. Section 5 is devoted the description of several numerical experiments. Conclusions are offered in Section 6 .

2. The model. The physical quantities and corresponding equilibrium equations are expressed in spherical polar coordinates, with the origin corresponding to the vertex of the hopper. For non-axisymmetric domains, coordinate systems that are better suited to the geometry at hand can usually be constructed. However, such systems are typically not orthogonal, complicating greatly the structure of the basic equations of Continuum Mechanics [1]. To simplify the numerics, such alternate coordinate systems are introduced in Section 4 through a change of variables, but the individual components of the stress tensor, velocity, etc..., are still measured in terms in the original spherical coordinates.

The strain rate tensor $V=-\frac{1}{2}\left(\nabla v+\nabla v^{T}\right), v$ being the velocity, and the stress tensor $T$ take respectively the form (see e.g.[19], p.184) ${ }^{1}$

$$
\begin{gathered}
V=\left[\begin{array}{ccc}
-\partial_{r} v_{r} & -\frac{1}{2}\left(\frac{1}{r} \partial_{\theta} v_{r}-\frac{v_{\theta}}{r}+\partial_{r} v_{\theta}\right) & -\frac{1}{2}\left(\frac{1}{r \sin \theta} \partial_{\phi} v_{r}-\frac{v_{\phi}}{r}+\partial_{r} v_{\phi}\right) \\
\cdot & -\frac{1}{r}\left(v_{r}+\partial_{\theta} v_{\theta}\right) & -\frac{1}{2 r}\left(\partial_{\theta} v_{\phi}-\cot \theta v_{\phi}+\frac{1}{\sin \theta} \partial_{\phi} v_{\theta}\right) \\
\cdot & \cdot \frac{1}{r}\left(v_{r}+\cot \theta v_{\theta}+\frac{1}{\sin \theta} \partial_{\phi} v_{\phi}\right)
\end{array}\right] \\
T=\left[\begin{array}{ccc}
T_{r r} & T_{r \theta} & T_{r \phi} \\
\cdot & T_{\theta \theta} & T_{\theta \phi} \\
\cdot & \cdot & T_{\phi \phi}
\end{array}\right]
\end{gathered}
$$

\footnotetext{
${ }^{1}$ We omit to write the lower triangular part of symmetric tensors.
} 
The equations of equilibrium are

$$
\nabla \cdot T=\rho \mathbf{g},
$$

where $\mathbf{g}$ is the acceleration vector due gravity, or equivalently

$$
\begin{aligned}
\partial_{r} T_{r r}+\frac{1}{r \sin \theta} \partial_{\phi} T_{r \phi}+\frac{1}{r} \partial_{\theta} T_{r \theta}+\frac{1}{r}\left(2 T_{r r}-T_{\phi \phi}-T_{\theta \theta}+T_{r \theta} \cot \theta\right) & =-\rho g \cos \theta, \\
\partial_{r} T_{r \phi}+\frac{1}{r \sin \theta} \partial_{\phi} T_{\phi \phi}+\frac{1}{r} \partial_{\theta} T_{\phi \theta}+\frac{1}{r}\left(3 T_{r \phi}+2 T_{\phi \theta} \cot \theta\right) & =0, \\
\partial_{r} T_{r \theta}+\frac{1}{r \sin \theta} \partial_{\phi} T_{\theta \phi}+\frac{1}{r} \partial_{\theta} T_{\theta \theta}+\frac{1}{r}\left(3 T_{r \theta}+\left(T_{\theta \theta}-T_{\phi \phi}\right) \cot \theta\right) & =\rho g \sin \theta,
\end{aligned}
$$

where $g=|\mathbf{g}|$ and $\rho$ is the density.

For the plasticity model, the von Mises yield condition is assumed to hold. Expressed in terms of the principal stresses, $\sigma_{i}, \mathrm{i}=1,2,3$, i.e., the eigenvalues of $T$, this condition reads

$$
\left(\sigma_{1}-p\right)^{2}+\left(\sigma_{2}-p\right)^{2}+\left(\sigma_{3}-p\right)^{2}=2 p^{2} s^{2}
$$

where

$$
p=\frac{1}{3} \operatorname{tr} T=\frac{1}{3}\left(T_{r r}+T_{\theta \theta}+T_{\phi \phi}\right)=\frac{1}{3}\left(\sigma_{1}+\sigma_{2}+\sigma_{3}\right)
$$

is the average stress and $s=\sin \delta, \delta$ being the angle of internal friction.

A flow rule completes the model. The eigenvectors of the strain rate tensor $V$ and the deviatoric part of the stress tensor $T$ are assumed to be parallel. This is the Levy flow rule, which can be equivalently expressed as the existence of a positive scalar function $\lambda>0$ such that

$$
V=\lambda(T-p I)
$$

The alignment condition of the eigenvectors of $T$ and $V$ in effect neglects the rotation of a material element during deformation, a controversial assumption. There is experimental evidence that misalignment may occur under some circumstances. Alternative models which allow for the above eigenvectors to be somewhat out of alignment have been proposed, see e.g. [20]. However, to the best of our knowledge, there does not seem to be enough experimental data to favor one type of models over the other. We refer to [7] for a lucid, if somewhat dated, account of the situation. Further, and more importantly, the fact that we look here exclusively at radial flows, renders the distinction between the two types of model less of an issue. Indeed, simple explicit calculations show that in the case of radial flows in three dimensional conical hoppers of circular cross section, for instance, the misalignment predicted by Spencer's double shearing model [20] is less than $10^{\circ}$ in all cases of physical interest.

The unknowns characterizing the flow are the velocity $v$ and the stress tensor $T$, assuming constant density. They are determined by (2.1) which corresponds to conservation of momentum, the yield condition (2.2) and the above flow rule (2.3). Note that (2.3) also implies incompressibility, and thus conservation of mass, as

$$
\nabla \cdot v=-\operatorname{tr} V=\lambda \operatorname{tr}(T-p I)=0 .
$$

The type of the system (2.1-2.3), which has to be supplemented with side conditions discussed below, can be determined. In [17], it was shown that, depending among 
other things on the internal friction, the system for fully three-dimensional flows can be mixed hyperbolic-elliptic, elliptic or have no definite type.

Following Jenike [8], [9], we seek similarity solutions with radial symmetry. More precisely, the velocity field is assumed to be purely radial, i.e.

$$
\left[v_{r}, v_{\theta}, v_{\phi}\right]=\left[v_{r}, 0,0\right]
$$

while the stress components are of the form

$$
T_{r r}=r \tau_{r r}(\theta, \phi),
$$

and similarly for the other stress components. The strain rate tensor simplifies to

$$
V=\left[\begin{array}{ccc}
-\partial_{r} v_{r} & -\frac{1}{2 r} \partial_{\theta} v_{r} & -\frac{1}{2 r \sin \theta} \partial_{\phi} v_{r} \\
\cdot & -\frac{1}{r} v_{r} & 0 \\
\cdot & \cdot & -\frac{1}{r} v_{r}
\end{array}\right]
$$

The flow rule (2.3) has several fundamental consequences. First, since again $\nabla \cdot v=0$, we obtain

$$
\partial_{r} v_{r}+\frac{2}{r} v_{r}=0
$$

The proper scaling for the velocity is consequently

$$
v_{r}(r, \theta, \phi)=-\frac{1}{r^{2}} v(\theta, \phi)
$$

where we further have

$$
\partial_{\theta} v=\frac{2 T_{r \theta}}{T_{\theta \theta}-p} v
$$

Finally, (2.3) implies

$$
\tau_{\theta \theta}=\tau_{\phi \phi} \text { and } \tau_{\theta \phi}=0 .
$$

The yield condition (2.2) can be expressed in terms of the four stress unknowns $\tau_{r r}$, $\tau_{r \theta}, \tau_{r \phi}$ and $\tau_{\theta \theta}$ by using the invariants of $T$, see (2.8) below. This results in the following system of three partial differential equations and one algebraic constraint in the four remaining stress unknowns $\tau_{r r}, \tau_{r \theta}, \tau_{r \phi}$ and $\tau_{\theta \theta}$

$$
\begin{aligned}
\frac{1}{\sin \theta} \partial_{\phi} \tau_{r \phi}+\partial_{\theta} \tau_{r \theta}+3 \tau_{r r}-2 \tau_{\theta \theta}+\tau_{r \theta} \cot \theta & =-\rho g \cos \theta \\
\frac{1}{\sin \theta} \partial_{\phi} \tau_{\theta \theta}+4 \tau_{r \phi} & =0 \\
\partial_{\theta} \tau_{\theta \theta}+4 \tau_{r \theta} & =\rho g \sin \theta \\
\left(\tau_{r r}-\tau_{\theta \theta}\right)^{2}+3 \tau_{r \theta}^{2}+3 \tau_{r \phi}^{2} & =\frac{1}{3}\left(\tau_{r r}+2 \tau_{\theta \theta}\right)^{2} s^{2} .
\end{aligned}
$$

The yield condition (2.8) deserves further comments. It can be written

$$
\mathcal{T}^{T} A \mathcal{T}=-3 \tau_{r \phi}^{2}, \quad \text { with } \mathcal{T}=\left[\begin{array}{c}
\tau_{r r} \\
\tau_{r \theta} \\
\tau_{\theta \theta}
\end{array}\right], \quad A=\left[\begin{array}{ccc}
1-\frac{s^{2}}{3} & 0 & -1-\frac{2 s^{2}}{3} \\
0 & 3 & 0 \\
-1-\frac{2 s^{2}}{3} & 0 & 1-\frac{4 s^{2}}{3}
\end{array}\right]
$$




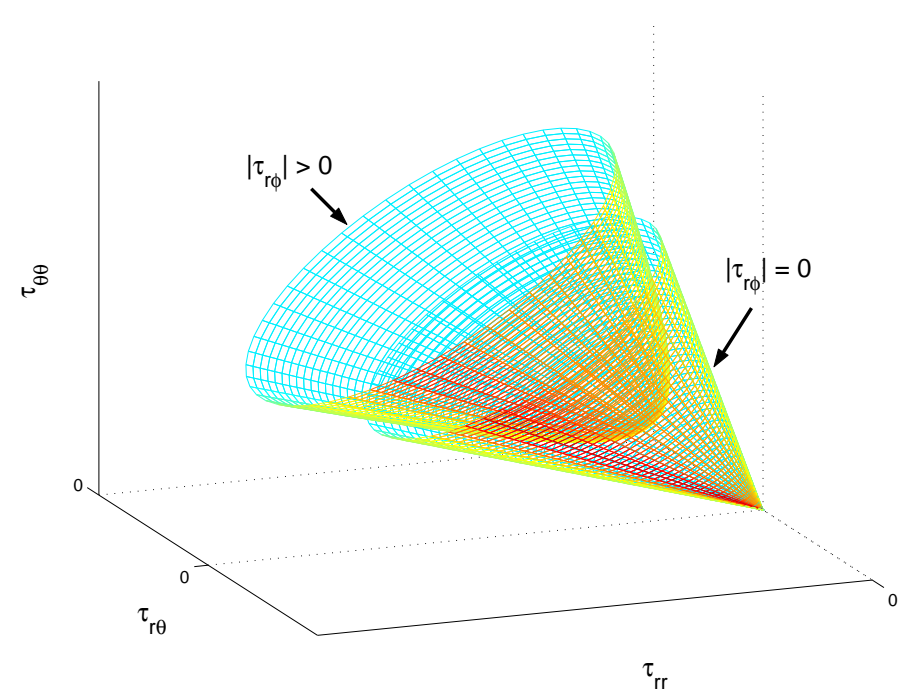

FIG. 2.1. Two representative level surfaces corresponding to the yield condition. In each case, only the physically relevant nappe is showed.

The symmetric matrix $A$ can be diagonalized

$$
A=X \Lambda X^{T}, \quad \text { with } \Lambda=\left[\begin{array}{lll}
\lambda_{1} & & \\
& \lambda_{2} & \\
& & \lambda_{3}
\end{array}\right], \quad X=\left[\begin{array}{l|l|l}
x_{1} & x_{2} & x_{3}
\end{array}\right],
$$

where

$$
\begin{aligned}
& \lambda_{1}=3, \quad \lambda_{2,3}=1-\frac{5 s^{2}}{6} \pm \sqrt{1+\frac{4}{3} s^{2}+\left(\frac{5 s^{2}}{6}\right)^{2}} \\
& x_{1}=[0,1,0]^{T}, \quad x_{2,3}=\left[-\frac{1}{3+2 s^{2}}\left(\frac{3 s^{2}}{2} \pm \frac{1}{2} \sqrt{36+48 s^{2}+25 s^{4}}\right), 0,1\right]^{T} .
\end{aligned}
$$

As is easily seen from those expressions, $\lambda_{1}$ and $\lambda_{2}$ are positive for any $\delta>0$, while $\lambda_{3}$ is negative. Let $\Xi=\left[\begin{array}{lll}\xi & \eta & \zeta\end{array}\right]^{T}=X^{T} \mathcal{T}$; the yield condition takes the form

$$
\Xi^{T} \Lambda \Xi \equiv 3 \xi^{2}+\lambda_{2} \eta^{2}+\lambda_{3} \zeta^{2}=-3 \tau_{r \phi}^{2},
$$

which corresponds to a family of hyperboloids of two sheets parametrized by $\left|\tau_{r \phi}\right|$, see Figure 2.1. The case $\tau_{r \phi}=0$ is a cone. Only one of the two sheets is physically relevant. Indeed, granular materials can only support compressive stresses, i.e., $\sigma_{i}>$ $0, i=1,2,3$. This condition is satisfied on only one of the sheets, provided $\delta<60^{\circ}$. The corresponding result given below can be found in [17] where it is stated and proved using exclusively the properties of the principal stresses (i.e., (2.2) instead of $(2.8))$.

Lemma 2.1. For any value of $\tau_{r \phi}$, the hyperboloid of two sheets (2.8) has one sheet corresponding to compressive stresses, $\sigma_{i}>0, i=1,2,3$, if and only if the internal angle of friction satisfies $0^{\circ}<\delta<60^{\circ}$. 
Proof. As a direct consequence of the structure of the scaled stress tensor $\tilde{T}=$ $\left[\begin{array}{ccc}\tau_{r r} & \tau_{r \theta} & \tau_{r \phi} \\ \tau_{r \theta} & \tau_{\theta \theta} & 0 \\ \tau_{r \phi} & 0 & \tau_{\theta \theta}\end{array}\right]$, it can be seen that the principal stresses are

$$
\begin{aligned}
\tilde{\sigma}_{1} & =\tau_{\theta \theta}, \\
\tilde{\sigma}_{2,3} & =\frac{\tau_{\theta \theta}+\tau_{r r}}{2} \pm \sqrt{\left(\tau_{\theta \theta}-\tau_{r r}\right)^{2}+4 \tau_{r \theta}^{2}+4 \tau_{r \phi}^{2}},
\end{aligned}
$$

without assuming any ordering of the $\tilde{\sigma}_{i}$ 's. The component $\tau_{\theta \theta}$ has thus to be positive. Further, we have

$$
\operatorname{det} \tilde{T}=\tau_{\theta \theta}\left(\tau_{r r} \tau_{\theta \theta}-\tau_{r \theta}^{2}-\tau_{r \phi}^{2}\right)=\tilde{\sigma}_{1} \tilde{\sigma}_{2} \tilde{\sigma}_{3}
$$

and thus

$$
\tau_{r r} \tau_{\theta \theta}-\tau_{r \theta}^{2}-\tau_{r \phi}^{2}=\tilde{\sigma}_{2} \tilde{\sigma}_{3},
$$

Therefore, $\tilde{\sigma}_{2}$ and $\tilde{\sigma}_{3}$ only have the same sign if $\tau_{r r}>0$. Solving for $\tau_{r r}$ directly from the yield condition (2.8) shows that $\tau_{r r}>0$ for positive values of $\tau_{\theta \theta}$ provided $2 s^{2}-3 \sqrt{3} s+3>0$, see (2.13) below. Since $s=\sin \delta$, it is equivalent to $\delta<60^{\circ}$.

if

Combining (2.8) with (2.11), we observe that the remaining $\tilde{\sigma}_{2}$ and $\tilde{\sigma}_{3}$ are positive

$$
\left(\frac{s^{2}}{3}-1\right) \tau_{r r}^{2}+\left(\frac{4 s^{2}}{3}-1\right) \tau_{\theta \theta}^{2}+\left(\frac{4 s^{2}}{3}+2\right) \tau_{r r} \tau_{\theta \theta}<3 \tau_{r r} \tau_{\theta \theta},
$$

which is clearly satisfied if again $\delta<60^{\circ}$.

At this point, the system (2.5-2.8) can be rewritten in several ways. One could for instance introduce à la Sokolovskii variables [14] which essentially would correspond to solving (2.9) by replacing $\xi$ and $\eta$ by one new variable $\psi$

$$
\xi=-\sqrt{\frac{-1}{3}\left(\lambda_{3} \zeta^{2}+3 \tau_{r \phi}^{2}\right)} \sin 2 \psi, \quad \eta=\sqrt{\frac{-1}{\lambda_{2}}\left(\lambda_{3} \zeta^{2}+3 \tau_{r \phi}^{2}\right)} \cos 2 \psi .
$$

Note that the above expressions make sense because of the yield condition (2.9) and the fact that $\lambda_{2}>0$. The above approach would lead to an elliptic system of three fully nonlinear first order equations in the variables $\zeta, \psi$ and $\tau_{r \phi}$. We choose rather to work with the original scaled variables after having solved (2.8) for $\tau_{r r}$

$$
\begin{aligned}
\tau_{r r} & =f_{ \pm}\left(\tau_{r \theta}, \tau_{r \phi}, \tau_{\theta \theta}\right) \\
& \equiv \frac{1}{3-s^{2}}\left(\left(3+2 s^{2}\right) \tau_{\theta \theta} \pm 3 \sqrt{3 s^{2} \tau_{\theta \theta}^{2}-\left(3-s^{2}\right) \tau_{r \theta}^{2}-\left(3-s^{2}\right) \tau_{r \phi}^{2}}\right) .
\end{aligned}
$$

As can obviously be seen from both the above expression and Figure 2.1, for any triple $\left(\tau_{r \theta}, \tau_{r \phi}, \tau_{\theta \theta}\right)$, one can have zero, one or two corresponding values of $\tau_{r r}$. The zero solution case, i.e.

$$
\tau_{\theta \theta}^{2}<\frac{3-s^{2}}{3 s^{2}}\left(\tau_{r \theta}^{2}+\tau_{r \phi}^{2}\right),
$$

corresponds, by assumption here, to stress states incompatible with the physical properties of the material under consideration. 
The two solutions in (2.13) are related to the so-called active (+ sign) and passive (- sign) states of the flowing material, see e.g. [14]. As the passive state is the one that tends to be observed experimentally upon discharge of the hopper, we adopt, to fix the ideas, $\tau_{r r}=f_{-}\left(\tau_{r \theta}, \tau_{r \phi}, \tau_{\theta \theta}\right)$. However, the analysis presented below applies equally to both cases.

The unknowns $\tau_{r \phi}$ and $\tau_{r \theta}$ can be eliminated from the system by using (2.6) and (2.7). Relation (2.5) becomes

$$
-\frac{1}{\sin ^{2} \theta} \partial_{\phi \phi} \tau_{\theta \theta}-\frac{1}{\sin \theta} \partial_{\theta}\left(\sin \theta \partial_{\theta} \tau_{\theta \theta}\right)+12 \tau_{r r}-8 \tau_{\theta \theta}=-6 \rho g \cos \theta .
$$

One can recognize the above differential operator $-\frac{1}{\sin ^{2} \theta} \partial_{\phi \phi} \cdot-\frac{1}{\sin \theta} \partial_{\theta}\left(\sin \theta \partial_{\theta} \cdot\right)=-\Delta$ as Laplace's operator on the sphere. The system is closed by expressing $\tau_{r r}$ as a function of $\tau_{\theta \theta}$ and its first derivatives through (2.6), (2.7) and (2.13), leading to

$$
-\Delta \tau_{\theta \theta}+\chi \tau_{\theta \theta}=-6 \rho g \cos \theta+F\left(\theta, \tau_{\theta \theta}, \partial_{\theta} \tau_{\theta \theta}, \partial_{\phi} \tau_{\theta \theta}\right),
$$

where $\chi=4 \frac{3+8 s^{2}}{3-s^{2}}$ and

$F\left(\theta, \tau_{\theta \theta}, \partial_{\theta} \tau_{\theta \theta}, \partial_{\phi} \tau_{\theta \theta}\right)=\frac{9}{3-s^{2}} \sqrt{48 s^{2} \tau_{\theta \theta}^{2}-\left(3-s^{2}\right)\left(\left(\rho g \sin \theta-\partial_{\theta} \tau_{\theta \theta}\right)^{2}+\left(\frac{\partial_{\phi} \tau_{\theta \theta}}{\sin \theta}\right)^{2}\right)}$.

The above nonlinear Helmholtz equation (2.14) has to be solved in a subdomain $\Omega$ of the sphere corresponding to a "triangle", with two edges corresponding to arcs of great circles and a third one corresponding to the outer boundary, see Figure 2.2 in the case of a domain with rectangular cross section. The boundary $\partial \Omega$ of $\Omega$ consists of the four parts

$$
\partial \Omega=\Gamma_{0} \cup \Gamma_{1} \cup \Gamma_{2} \cup \Gamma_{3},
$$

where $\Gamma_{0}$ is the trivial boundary $\{(\theta, \phi) ; \theta=0\}$ and $\Gamma_{1,3}=\left\{(\theta, \phi) ; 0<\theta<\theta_{w}, \phi=\right.$ $\left.\mp \phi_{w}\right\}$, with $\theta_{w}>0$ and $\phi_{w}>0$. The boundary $\Gamma_{2}$ is represented as the graph of an even function $\mathcal{C}$ of class $C^{1}, \theta_{w}=\mathcal{C}\left( \pm \phi_{w}\right)$

$$
\Gamma_{2}=\left\{(\theta, \phi) ; \theta=\mathcal{C}(\phi),-\phi_{w}<\phi<\phi_{w}\right\} .
$$

The domain $\Omega$ in which the problem is to be solved is thus

$$
\Omega=\left\{(\theta, \phi) ;-\phi_{w}<\phi<\phi_{w}, 0<\theta<\mathcal{C}(\phi)\right\},
$$

see Figure 2.2.

The boundary conditions are derived from physical considerations. On $\Gamma_{1}$ and $\Gamma_{3}$, we impose

$$
\tau_{\theta \theta}\left(\cdot,-\phi_{w}\right)=\tau_{\theta \theta}\left(\cdot, \phi_{w}\right), \quad \partial_{\phi} \tau_{\theta \theta}\left(\cdot,-\phi_{w}\right)=\partial_{\phi} \tau_{\theta \theta}\left(\cdot, \phi_{w}\right)=0 .
$$

Indeed, by symmetry, it is clear that one should have

$$
\tau_{\theta \theta}\left(\cdot,-\phi_{w}\right)=\tau_{\theta \theta}\left(\cdot, \phi_{w}\right), \quad \partial_{\phi} \tau_{\theta \theta}\left(\cdot,-\phi_{w}\right)=-\partial_{\phi} \tau_{\theta \theta}\left(\cdot, \phi_{w}\right) .
$$

Further, by a formal regularity argument, one obtains that $\partial_{\phi} \tau_{\theta \theta}$ vanishes on $\Gamma_{1}$ and $\Gamma_{3}$. Indeed, consider the problem in the full domain $\Omega_{\text {full }}=\{(\theta, \phi) ; 0<\theta<$ 

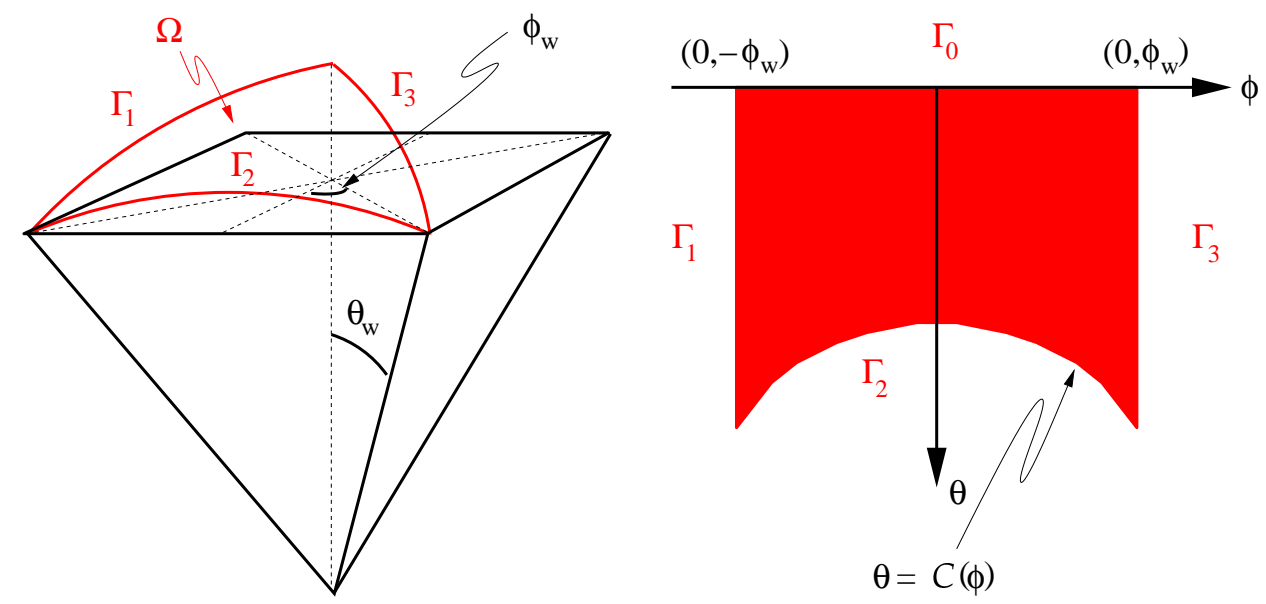

FIG. 2.2. Geometry of the computational domain.

$\left.\mathcal{C}_{\text {per }}(\phi), \phi \in[0,2 \pi]\right\}$, where $\mathcal{C}_{\text {per }}(\phi)$ is constructed from $\mathcal{C}(\phi)$ by using the periodicity of the entire hopper section. Freezing the coefficients in the nonlinear part $F$ of (2.14), classic regularity results, see [6] $\S 3.2$ and Remark 3.2.4.6 yield $H^{2}$-regularity of the solutions. For such a solution $u$, one has on each $\phi=c s t$ line $\Gamma_{\phi}$

$$
\gamma \partial_{\phi} u^{+}=\gamma \partial_{\phi} u^{-}
$$

where $u^{ \pm}$correspond to the values from each side of $\Gamma_{\phi}$ and the mapping $\gamma: H^{2}\left(\Omega_{\text {full }}\right) \rightarrow$ $H^{1 / 2}\left(\Gamma_{\phi}\right)$ which takes $u$ to $\gamma \partial_{\phi} u$ is the normal trace operator, see [6], Lemma 1.5.1.8.

The condition $\partial_{\phi} \tau_{\theta \theta}\left( \pm \phi_{w}\right)=0$ has also been verified numerically by varying the domain of resolution (for instance, by rotating it by $\pi / 4$ in case of a square domain).

On $\Gamma_{2}$, the law of sliding friction applies, i.e.

$$
\left|\tilde{T}_{T}\right|=\mu_{w} \tilde{T}_{N}, \quad \text { on } \Gamma_{2},
$$

where $\tilde{T}_{T}$ and $\tilde{T}_{N}$ are the scaled tangential and normal stresses on the hopper wall, and $\mu_{w}$ is the coefficient of wall friction. Since the outer unit normal to the wall is

$$
N=\frac{\left[0, \sin \theta,-\mathcal{C}^{\prime}(\phi)\right]}{\sqrt{\sin ^{2} \theta+\mathcal{C}^{\prime}(\phi)^{2}}},
$$

it follows that

$$
\tilde{T}_{N}=\tau_{\theta \theta} \quad \text { and } \quad \tilde{T}_{T}=\left[N_{\theta} \tau_{r \theta}+N_{\phi} \tau_{r \phi}, 0,0\right]^{T} .
$$

The boundary condition on $\Gamma_{2}$ then reads by (2.6), (2.7)

$$
\begin{aligned}
\rho g \sin ^{2} \mathcal{C}(\phi)-\sin \mathcal{C}(\phi) \partial_{\theta} \tau_{\theta \theta}+ & \frac{\mathcal{C}^{\prime}(\phi)}{\sin \mathcal{C}(\phi)} \partial_{\phi} \partial_{\phi} \tau_{\theta \theta}= \\
& -4 \mu_{w} \sqrt{\sin ^{2} \mathcal{C}(\phi)+\mathcal{C}^{\prime}(\phi)^{2}} \tau_{\theta \theta}
\end{aligned}
$$

which is essentially a Robin boundary condition. 
3. Mathematical analysis. The above problem presents several nonstandard features and difficulties (Laplace-Beltrami operator, Robin boundary condition, non Lipschitz coefficients, restricted range of admissible values). In this section, an existence and local uniqueness result is established.

We equip $L^{2}(\Omega)$ with the inner product $(u, v)=\int_{-\phi_{w}}^{\phi_{w}} \int_{0}^{\mathcal{C}(\phi)} u v \sin \theta d \theta d \phi$. Let

$$
\begin{aligned}
& V=\left\{v \in L^{2}(\Omega) ; \nabla v\right. \equiv\left[\partial_{\theta} v, \frac{1}{\sin \theta} \partial_{\phi} v\right] \in L^{2}(\Omega)^{2} \\
&\left.u\left(\cdot,-\phi_{w}\right)=u\left(\cdot, \phi_{w}\right) \text { in the sense of the trace }\right\}
\end{aligned}
$$

The space $V$ is equipped with inner product and norm

$$
(u, v)_{V}=(u, v)+(\nabla u, \nabla v), \quad\|u\|_{V}=\sqrt{(u, u)_{V}} .
$$

Formally, if $u$ stands for a classical solution to problem (2.14) and if $v \in V$, we obtain after integration by parts and use of the boundary conditions (2.15) and (2.16)

$$
\begin{array}{rl}
-\iint_{\Omega} \Delta u & v d \omega=\int_{-\phi_{w}}^{\phi_{w}} \int_{0}^{\mathcal{C}(\phi)} \nabla u \cdot \nabla v \sin \theta d \theta d \phi \\
& -\int_{-\phi_{w}}^{\phi_{w}}\left(4 \mu_{w} \sqrt{\sin ^{2} \mathcal{C}(\phi)+\mathcal{C}^{\prime}(\phi)^{2}} u(\mathcal{C}(\phi), \phi)+\rho g \sin ^{2} \mathcal{C}(\phi)\right) v(\mathcal{C}(\phi), \phi) d \phi .
\end{array}
$$

Let $a: V \times V \rightarrow \mathrm{R}$ be the bilinear form defined by

$$
\begin{aligned}
a(u, v) & =\int_{-\phi_{w}}^{\phi_{w}} \int_{0}^{\mathcal{C}(\phi)}\{\nabla u \cdot \nabla v+\chi u v\} \sin \theta d \theta d \phi \\
& -4 \mu_{w} \int_{-\phi_{w}}^{\phi_{w}} \sqrt{\sin ^{2} \mathcal{C}(\phi)+\mathcal{C}^{\prime}(\phi)^{2}} u(\mathcal{C}(\phi), \phi) v(\mathcal{C}(\phi), \phi) d \phi .
\end{aligned}
$$

For $\Gamma_{2}$, i.e., $\mathcal{C}$ of class $C^{1}$, it is well known that the trace operator $\gamma: V \rightarrow H^{1 / 2}\left(\Gamma_{2}\right)$ is linear and continuous [6], Th. 1.5.1.3, p.38. Consequently, the form $a: V \times V \rightarrow \mathrm{R}$ is continuous. Further, under a condition that links wall friction, internal friction and the geometry of the domain (a precise condition is given as part of the proof of the next result), the bilinear form $a(\cdot, \cdot)$ is $V$-elliptic.

Proposition 3.1. The bilinear form a $(\cdot, \cdot)$ is $V$-elliptic, provided $\Gamma_{2}$ is a curve of class $C^{1}$ and $\mu_{w}$ is sufficiently small.

Proof. Let $e_{\theta}=[1,0]$ and $h(\phi)=\sqrt{\sin ^{2} \mathcal{C}(\phi)+\mathcal{C}^{\prime}(\phi)^{2}}$; we have

$$
\iint_{\Omega} h(\phi) \nabla\left(u^{2}\right) \cdot e_{\theta} d \omega=2 \iint_{\Omega} h(\phi) u \partial_{\theta} u d \omega .
$$

On the other hand, the divergence theorem leads to

$$
\begin{aligned}
\iint_{\Omega} h(\phi) \nabla\left(u^{2}\right) \cdot e_{\theta} d \omega & =-\iint_{\Omega} u^{2} \nabla \cdot\left(h(\phi) e_{\theta}\right) d \omega+\int_{\partial \Omega} h(\phi) u^{2} e_{\theta} \cdot N d \sigma \\
& =\int_{\Gamma_{2}} h(\phi) u^{2} e_{\theta} \cdot N d \sigma
\end{aligned}
$$


where $N$ stands again for the unit outer normal and where we have used (2.15). On $\Gamma_{2}$, we observe that

$$
h(\phi) e_{\theta} \cdot N=\sqrt{\sin ^{2} \mathcal{C}(\phi)+\mathcal{C}^{\prime}(\phi)^{2}} e_{\theta} \cdot N=\sin \mathcal{C}(\phi), \quad \phi \in\left[-\phi_{w}, \phi_{w}\right],
$$

and thus

$$
\int_{\Gamma_{2}} h(\phi) u^{2} e_{\theta} \cdot N d \sigma \geq \frac{\sin \theta_{\min }}{\sqrt{\sin ^{2} \theta_{\max }+\mathcal{C}_{\infty}^{\prime 2}}} \int_{\Gamma_{2}} h(\phi) u^{2} d \sigma
$$

where $\theta_{\min (\max )}=\min _{\phi \in\left[-\phi_{w}, \phi_{w}\right]}(\max ) \mathcal{C}(\phi)$ and $\mathcal{C}_{\infty}^{\prime}=\max _{\phi \in\left[-\phi_{w}, \phi_{w}\right]}\left|\mathcal{C}^{\prime}(\phi)\right|$. Combining the last three expressions and using $d \sigma=\sin \theta d \phi$ yields, for any $\eta>0$

$$
\begin{aligned}
\int_{-\phi_{w}}^{\phi_{w}} h(\phi) u^{2}(\mathcal{C}(\phi), \phi) d \phi & \leq 2 \frac{\sqrt{\sin ^{2} \theta_{\max }+\mathcal{C}_{\infty}^{\prime 2}}}{\sin ^{2} \theta_{\min }} \iint_{\Omega}|h(\phi)||u|\left|\partial_{\theta} u\right| d \omega \\
& \leq \frac{\sin ^{2} \theta_{\max }+\mathcal{C}_{\infty}^{\prime 2}}{\sin ^{2} \theta_{\min }}\left(\frac{1}{\eta}\|u\|_{L^{2}(\Omega)}^{2}+\eta\left\|\partial_{\theta} u\right\|_{L^{2}(\Omega)}^{2}\right) .
\end{aligned}
$$

From the definition of $a(\cdot, \cdot)$, sufficient conditions of $V$-ellipticity are then found to be

$$
\chi-\frac{4 \mu_{w}}{\eta} \frac{\sin ^{2} \theta_{\max }+\mathcal{C}_{\infty}^{\prime 2}}{\sin ^{2} \theta_{\min }}>0 \quad 1-4 \mu_{w} \eta \frac{\sin ^{2} \theta_{\max }+\mathcal{C}_{\infty}^{\prime 2}}{\sin ^{2} \theta_{\min }}>0
$$

for some $\eta>0$. A sufficient condition is then for instance

$$
\chi-32 \mu_{w}^{2}\left(\frac{\sin ^{2} \theta_{\max }+\mathcal{C}_{\infty}^{\prime 2}}{\sin ^{2} \theta_{\min }}\right)^{2}>0 .
$$

We can now state a weak formulation of our problem: Find a function $u \in V$ such that

$$
a(u, v)=\iint_{\Omega} F(u) v d \omega-6 \rho g \iint_{\Omega} \cos \theta v d \omega+\rho g \int_{\phi_{w}}^{\phi_{w}} \sin ^{2} \mathcal{C}(\phi) v(\mathcal{C}(\phi), \phi) d \phi,
$$

for any $v \in V$ and where, with a slight abuse of notation, $F$ still denotes the nonlinear function (2.14). We define $A \in \mathcal{L}\left(V, V^{\star}\right)$ and $\Phi \in V^{\star}$ by respectively

$$
\begin{aligned}
<A u, v> & =a(u, v), \quad \forall u, v \in V, \\
<\Phi, v> & =-6 \rho g \iint_{\Omega} \cos \theta v d \omega+\rho g \int_{-\phi_{w}}^{\phi_{w}} \sin ^{2} \mathcal{C}(\phi) v(\mathcal{C}(\phi), \phi) d \phi, \quad \forall v \in V,
\end{aligned}
$$

where $<\cdot, \cdot>$ denotes the $V, V^{\star}$-duality product. Identifying in the obvious way $F(u)$ with an element of $V^{\star}$, the problem becomes: Find $u \in V$ such that

$$
A u=F(u)+\Phi
$$

Let us denote by $\mathcal{G}: V \rightarrow V^{\star}$ the mapping

$$
\mathcal{G}(u)=A u-F(u)
$$


and let $u_{0}=-\rho g \cos \theta \in V$. Note that $\mathcal{G}\left(u_{0}\right) \in V^{\star}$ is well defined as $F\left(u_{0}\right)$ is itself well defined by construction, provided $\theta_{\max }<\pi / 2$. Direct calculations show

$$
\mathcal{L}\left(V, V^{\star}\right) \ni D \mathcal{G}(u)=A-D F(u),
$$

where

$$
<D F(u) h, v>=\frac{9}{3-s^{2}} \iint_{\Omega} \frac{48 s^{2} u h-\left(3-s^{2}\right)\left[-\left(\rho g \sin \theta-\partial_{\theta} u\right) \partial_{\theta} h+\frac{\partial_{\phi} u \partial_{\theta} h}{\sin ^{2} \theta}\right]}{\sqrt{48 s^{2} u^{2}-\left(3-s^{2}\right)\left[\left(\rho g \sin \theta-\partial_{\theta} u\right)^{2}+\left(\frac{\partial_{\phi} u}{\sin \theta}\right)^{2}\right]}} v d \omega,
$$

for any $h, v \in V$.

Proposition 3.2. Under the assumptions of Proposition 3.1, there are neighborhoods $U \subset V$ and $U^{\star} \subset V^{\star}$ of $u_{0}$ and $\mathcal{G}\left(u_{0}\right)$ respectively such that for each $\Phi \in U^{\star}$, the functional equation (3.1) has one and only one solution in $U$.

Proof. From the previous expression and the Inverse Mapping Theorem, $\mathcal{G}$ is a $C^{1}$-diffeomorphism between a neighborhood of $u_{0}$ in $V$ and one of $\mathcal{G}\left(u_{0}\right)$ in $V^{\star}$. The proposition follows.

Arguments following the lines of the formal remarks at the end of $\S 3$ could be used to obtain $H^{2}(\Omega)$-regularity of the solutions. We do not pursue this issue further here.

4. Numerical analysis. In order to simplify the numerics, the problem is now mapped onto a simple rectangular computational domain. From now on, the function $\mathcal{C}$ which describes $\Gamma_{2}$ is assumed to be of class $C^{2}$. We define the new coordinates

$$
\Theta=\theta_{w} \frac{\theta}{\mathcal{C}(\phi)} \quad \text { and } \quad \Phi=\phi
$$

Note that $\{r, \Theta, \Phi\}$ is not an orthogonal coordinate system. Keeping in mind that $\theta=\Theta \mathcal{C}(\phi) / \theta_{w}$, the problem for the transformed unknown $U(\Theta, \Phi)=u(\theta, \phi)$ takes the form

$$
\begin{aligned}
-\partial_{\Phi \Phi} U & +2 \Theta \frac{\mathcal{C}^{\prime}(\Phi)}{\mathcal{C}(\Phi)} \partial_{\Theta \Phi} U-\frac{1}{\mathcal{C}(\Phi)^{2}}\left(\Theta^{2} \mathcal{C}^{\prime}(\Phi)^{2}+\theta_{w}^{2} \sin ^{2} \theta\right) \partial_{\Theta \Theta} U \\
& +\left(\frac{\Theta}{\mathcal{C}^{2}(\Phi)}\left[\mathcal{C}^{\prime \prime}(\Phi) \mathcal{C}(\Phi)-2 \mathcal{C}^{\prime}(\Phi)^{2}\right]-\frac{1}{2} \frac{\theta_{w} \sin 2 \theta}{\mathcal{C}(\Phi)}\right) \partial_{\Theta} U+\chi \sin ^{2} \theta U \\
(4.1) & =-6 \rho g \cos \theta \sin ^{2} \theta+\mathcal{F}\left(\Theta, \Phi, U, \partial_{\Theta} U, \partial_{\Phi} U\right), \quad(\Theta, \Phi) \in\left(0, \theta_{w}\right) \times\left(-\phi_{w}, \phi_{w}\right),
\end{aligned}
$$

where

$\mathcal{F}=\frac{9 \sin \theta}{3-s^{2}} \sqrt{48 s^{2} \sin ^{2} \theta U^{2}-\left(3-s^{2}\right)\left(\left[\rho g \sin ^{2} \theta-\frac{\theta_{w} \sin \theta}{\mathcal{C}(\Phi)} \partial_{\Theta} U\right]^{2}+\left[\partial_{\Phi} U-\frac{\Theta \mathcal{C}^{\prime}(\Phi)}{\mathcal{C}(\Phi)} \partial_{\Theta} U\right]^{2}\right)}$.

The boundary condition on $\Gamma_{2}$ becomes

$$
\begin{aligned}
\rho g \sin ^{2} \mathcal{C}(\Phi)-\sin \mathcal{C}(\Phi) \frac{\theta_{w}}{\mathcal{C}(\Phi)} \partial_{\Theta} U+ & \frac{\mathcal{C}^{\prime}(\Phi)}{\sin \mathcal{C}(\Phi)}\left(-\theta_{w} \frac{\mathcal{C}^{\prime}(\Phi)}{\mathcal{C}(\Phi)} \partial_{\Theta}+\partial_{\Phi}\right) U= \\
& -4 \mu_{w} \sqrt{\sin ^{2} \mathcal{C}(\Phi)+\mathcal{C}^{\prime}(\Phi)^{2}} U, \quad \Phi \in\left(-\phi_{w}, \phi_{w}\right) .
\end{aligned}
$$

The above problem is discretized by collocation; Chebyshev collocation at the Chebyshev-Gauss-Radau points in used in $\Theta$, while Fourier-cosine collocation at the 
Fourier collocation points is used in $\Phi$. More precisely, we set

$$
U_{N M}(\Theta, \Phi)=\sum_{n=0}^{N-1} \sum_{m=-M / 2}^{M / 2-1} U_{n m} \psi_{n}(\Theta) e^{i m(4 \Phi+\pi)},
$$

where $\left\{\psi_{n}\right\}_{n=0}^{N-1}$ are the Lagrange interpolation polynomials at the Chebyshev-GaussRadau nodes on $\left[0, \theta_{w}\right]$, i.e.

$$
\Theta_{j}=\frac{\theta_{w}}{2}\left(1+\cos \left(\frac{2 \pi j}{2 N-1}\right)\right), \quad j=0, \ldots, N-1 .
$$

This choice, as opposed to the more standard Chebyshev-Gauss-Lobatto collocation, see e.g. [2], §2.4, results from the nature of the boundary condition along $\Theta=\theta_{w}$. For completeness, we derive the expression of the collocation derivative below (which we have not been able to find in the literature).

Lemma 4.1. The Lagrange interpolation polynomials on the Chebyshev-GaussRadau nodes (4.4) are given by

$\psi_{j}(\Theta)=\frac{1}{c_{j}} \frac{\theta_{w}-\Theta}{\Theta-\Theta_{j}}\left(\frac{1}{N} T_{N}^{\prime}\left(\frac{2 \Theta}{\theta_{w}}-1\right)+\frac{1}{N-1} T_{N-1}^{\prime}\left(\frac{2 \Theta}{\theta_{w}}-1\right)\right), \quad j=0, \ldots, N-1$

where

$$
\begin{aligned}
& c_{0}=1-2 N, \\
& c_{j}=-\frac{\theta_{w}}{2 \Theta_{j}}\left(N \cos \frac{2 \pi N j}{2 N-1}+(N-1) \cos \frac{2 \pi(N-1) j}{2 N-1}\right), \quad j=1, \ldots, N-1,
\end{aligned}
$$

where $T_{N}(x)=\cos (N \arccos x),|x| \leq 1$, is the Chebyshev polynomial of degree $N$.

The above result can easily be verified through the use of l'Hospital's rule and elementary properties of the Chebyshev polynomials. Interpolation at the nodes (4.4) of a function $u$ of $\Theta$ defined in $\left[0, \theta_{w}\right]$ simplify takes the form

$$
I_{N} u(\Theta)=\sum_{j=0}^{N-1} u\left(\Theta_{j}\right) \psi_{j}(\Theta)
$$

By definition, the Chebyshev collocation derivative of $u$ at those nodes is then

$$
\left(I_{N} u\right)^{\prime}\left(\Theta_{l}\right)=\sum_{j=0}^{N-1} u\left(\Theta_{j}\right) \psi_{j}^{\prime}\left(\Theta_{l}\right)=\sum_{j=0}^{N-1} \mathcal{D}_{l j} u\left(\Theta_{j}\right),
$$

with $\mathcal{D}_{l j}=\psi_{j}^{\prime}\left(\Theta_{l}\right)$. The collocation derivative at the nodes can then be obtained through matrix multiplication. Elementary albeit tedious calculations lead to the 
following expressions

$$
\mathcal{D}_{l j}= \begin{cases}\frac{2}{3} \frac{1}{\theta_{w}} N(N-1), & \text { if } l=j=0, \\ \frac{1}{c_{0} \theta_{w}} \frac{2}{\sin ^{2} \frac{2 \pi l}{2 N-1}}\left(N \cos \frac{2 N \pi l}{2 N-1}+(N-1) \cos \frac{2(N-1) \pi l}{2 N-1}\right) & \text { if } j=0, \\ & l=1, \ldots, N-1, \\ \frac{c_{l}}{c_{j}} \frac{1}{\Theta_{l}-\Theta_{j}} & \text { if } j=1, \ldots, N-1, \\ & j \neq l, l=0, \ldots, N-1, \\ -\frac{1}{4 \Theta_{j}} \frac{3 \theta_{w}-2 \Theta_{j}}{\theta_{w}-\Theta_{j}}-\frac{\theta_{w}}{4} \frac{1}{c_{j} \Theta_{j}} \frac{1}{\sqrt{\Theta_{j}\left(\theta_{w}-\Theta_{j}\right)}}\left(N^{2} \sin \left(N \arccos \left(\frac{2 \Theta_{j}}{\theta_{w}}-1\right)\right)+\right. \\ \left.(N-1)^{2} \sin \left((N-1) \arccos \left(\frac{2 \Theta_{j}}{\theta_{w}}-1\right)\right)\right) & \text { if } j=l=1, \ldots, N-1 .\end{cases}
$$

In the $\Phi$ direction, the collocation points are taken as the usual Fourier collocation nodes, i.e.,

$$
\Phi_{l}=\phi_{w}\left(\frac{2 l}{M}-1\right), \quad l=0, \ldots, M-1 .
$$

Let $\mathcal{U}$ be the $N \times M$ matrix of coefficients $U_{n m}, n=0, \ldots, N-1, m=0, \ldots, M / 2-$ $1,-M / 2, \ldots,-1$ and let $\mathcal{W}$ be the $M \times M$ Fourier matrix

$$
\mathcal{W}=\left[\begin{array}{lllll}
1 & 1 & 1 & \ldots & 1 \\
1 & \omega_{M} & \omega_{m}^{2} & \ldots & \omega_{M}^{M-1} \\
1 & \omega_{M}^{2} & \omega_{m}^{4} & \ldots & \omega_{M}^{2(M-1)} \\
\ldots & \ldots & \ldots & \ldots & \ldots \\
1 & \omega_{M}^{M-1} & \omega_{m}^{2(M-1)} & \ldots & \omega_{M}^{(M-1)^{2}}
\end{array}\right]
$$

where $\omega_{M}=e^{i 2 \pi / M}$ is the primitive $M$-th root of unity. Further, if $\mathcal{L}$ is the $M \times M$ diagonal matrix with diagonal $[0, \ldots, M / 2-1,-M / 2, \ldots,-1]$, then for any $j, l, j=$ $0, \ldots, N-1, l=0, \ldots, M-1$, the nodal values of $U_{N M}$ and its derivatives can be expressed as follows

$$
\begin{aligned}
U_{N M}\left(\Theta_{j}, \Phi_{l}\right) & =(\mathcal{U} \mathcal{W})_{j l}, \\
\partial_{\Phi} U_{N M}\left(\Theta_{j}, \Phi_{l}\right) & =4 i(\mathcal{U} \mathcal{L} \mathcal{W})_{j l}, \\
\partial_{\Phi \Phi} U_{N M}\left(\Theta_{j}, \Phi_{l}\right) & =-16\left(\mathcal{U} \mathcal{L}^{2} \mathcal{W}\right)_{j l}, \\
\partial_{\Theta} U_{N M}\left(\Theta_{j}, \Phi_{l}\right) & =(\mathcal{D U} \mathcal{W})_{j l}, \\
\partial_{\Theta \Phi} U_{N M}\left(\Theta_{j}, \Phi_{l}\right) & =4 i(\mathcal{D U} \mathcal{L} \mathcal{W})_{j l}, \\
\partial_{\Theta \Theta} U_{N M}\left(\Theta_{j}, \Phi_{l}\right) & =\left(\mathcal{D}^{2} \mathcal{U} \mathcal{W}\right)_{j l} .
\end{aligned}
$$

The $N \times M$ matrix of unknown coefficients $\mathcal{U}$ clearly satisfies a matrix equation of the type

$$
A_{1} \mathcal{U} B_{1}+\ldots A_{p} \mathcal{U} B_{p}=F(\mathcal{U})
$$

where the $A_{i}$ 's are $N \times N$ matrices while the $B_{i}$ 's are $M \times M$. The above system is obtained by enforcing the conditions that first, the discrete solution $U_{N M}$ from (4.3) satisfies the $\operatorname{PDE}(4.1)$ at the collocation points $\left\{\left(\Theta_{j}, \Phi_{l}\right)\right\}, j=1, \ldots, N-1$, 
$l=0, \ldots, M-1$ and second, that the boundary condition (4.2) is verified at the nodes $\left.\left\{\Theta_{0}, \Phi_{l}\right)\right\}, l=0, \ldots, M-1$. Note that no side conditions of any form are imposed in the neighborhood at $\Theta=0$. We denote by $A \otimes B$ the $N M \times N M$ matrix corresponding to the Kronecker product of an $N \times N$ matrix $A$ by an $M \times M$ matrix $B$. Further, for any matrix $A, \operatorname{vec}(A)$ denotes the vector formed by stacking the columns of $A$. One can check [12], p.410

$$
\operatorname{vec}(A \mathcal{U} B)=\left(B^{T} \otimes A\right) \operatorname{vec}(\mathcal{U}) .
$$

A direct consequence of this elementary relation is that the above matrix equation can be rewritten

$$
\mathbb{H} \operatorname{vec}(\mathcal{U})=\operatorname{vec}(F(\mathcal{U})),
$$

where $\mathbb{H}=\sum_{j=1}^{p}\left(B_{j}^{T} \otimes A_{j}\right)$ is a non sparse $N M \times N M$-matrix.

The above nonlinear system is numerically solved as follows. First, the matrix $\mathbb{H}$ is factorized into $\mathbb{H}=\mathbb{L} \mathbb{V}$, where $\mathbb{V}$ is an upper triangular matrix and $\mathbb{L}$ is a "psychologically" lower triangular matrix (LU factorization). Then, the nonlinear equation

$$
\operatorname{vec}(\mathcal{U})=\mathbb{V}^{-1} \mathbb{L}^{-1} \operatorname{vec}(F(\mathcal{U})),
$$

is solved by a Newton-GMRES solver [10].

5. Numerical results. The numerical approach is tested by comparing with an exact solution obtained for a simplified linear problem in a domain corresponding to a circular cone. More precisely, we consider the following problem

$$
\begin{aligned}
& -\frac{1}{\sin \theta}\left(\sin \theta u^{\prime}(\theta)\right)^{\prime}+\chi u=F \quad \text { for } 0<\theta<\theta_{w}, \\
& u^{\prime}(0)=0 \quad u^{\prime}\left(\theta_{w}\right)=\rho g \sin \theta_{w}+4 \mu_{w} u\left(\theta_{w}\right),
\end{aligned}
$$

where $F$ is taken as constant. The above equation and boundary conditions of this model problem corresponds exactly to $(4.1,4.2)$ whith a very simplified right-hand side.

The change of variable $v(z)=u(\theta)$ with $z=\cos \theta$ leads to

$$
\left(\left(1-z^{2}\right) v^{\prime}(z)\right)^{\prime}+\nu(\nu+1) v(z)=-F,
$$

where $\nu(\nu+1)=-\chi$. A fundamental system of solution to the homogeneous equation

$$
\left(\left(1-z^{2}\right) v^{\prime}(z)\right)^{\prime}+\nu(\nu+1) v(z)=0,
$$

is provided by the Legendre functions $P_{\nu}(z)$ and $Q_{\nu}(z)$ of the first and second kind respectively, see e.g. [3], $\S 8.82,8.83$. The coefficient $\nu$ defined by $\nu(\nu+1)=-\chi$ satisfies

$$
\nu=-\frac{1}{2} \pm \sqrt{\frac{1}{4}-\chi}=-\frac{1}{2} \pm i \lambda,
$$

where $\lambda=\sqrt{\chi-\frac{1}{4}}$ is a real parameter as, obviously, $\chi=4 \frac{3+8 s^{2}}{3-s^{2}} \geq 4$ for any material. We thus get the solution in terms of $P_{-\frac{1}{2}+i \lambda}(z)$ and $Q_{-\frac{1}{2}+i \lambda}(z)$ which are conical functions, see [3], §8.84. The general solution to (5.1) is then

$$
u(\theta)=A P_{-\frac{1}{2}+i \lambda}(\cos \theta)+B Q_{-\frac{1}{2}+i \lambda}(\cos \theta)+F / \chi,
$$




\begin{tabular}{||l|c|c|c|c||}
\hline$N=M$ & 8 & 10 & 12 & 14 \\
\hline error & $7.061(-8)$ & $1.723(-10)$ & $3.567(-13)$ & $5.995(-15)$ \\
\hline
\end{tabular}

TABLE 5.1

Maximum norm of the error for the model problem (5.1), (5.2).

where the last term is a particular solution to the considered problem. Since for real $\theta, Q_{-\frac{1}{2}+i \lambda}(\theta)$ is always imaginary while $P_{-\frac{1}{2}+i \lambda}$ is always real, we get $B=0$. After noticing that $u^{\prime}(0)=0$, the value of $A$ can be found from (5.2)

$$
A=\frac{\rho g \sin \theta_{w}+4 \mu_{w} F / \chi}{p^{\prime}\left(\theta_{w}\right)-4 \mu_{w} p\left(\theta_{w}\right)}, \quad \text { where } p(\theta)=P_{-\frac{1}{2}+i \lambda}(\cos \theta) .
$$

Our numerical solver is now applied to the present model problem (5.1), (5.2). The corresponding numerical solutions are compared to the exact solution (5.3). The results are summarized in Table 5.1, where the maximum norm of the error is given as a function of the number of nodes, taken here as $N=M$. This illustrates two facts. First, as expected, the method is found to be spectrally accurate. Second, for this simple problem, a mesh as small as $14 \times 14$ leads to errors of the order of the round-off errors.

The standard fully axisymmetric case of a circular cone for the full problem provides an additional way of testing the approach, this time in the nonlinear regime. Again, axisymmetry considerably simplifies the problem. Since no variable depends on $\phi$ in that case, a direct consequence of the flow rule (2.3) is that the (scaled) stress tensor $\tilde{T}$ reduces to

$$
\tilde{T}=\left[\begin{array}{ccc}
\tau_{r r} & \tau_{r \theta} & 0 \\
\tau_{r \theta} & \tau_{\theta \theta} & 0 \\
0 & 0 & \tau_{\theta \theta}
\end{array}\right]
$$

Further, the yield condition (2.8) reduces, in $\left(\tau_{r r}, \tau_{r \theta}, \tau_{\theta \theta}\right)$-space, to the cone illustrated in Figure $2.1\left(T_{r \phi}=0\right)$, as opposed to the family of hyperboloids discussed in $\S 2$. This cone can be parametrized by the Sokolovskii variables $(\sigma, \psi)$ where $\sigma$ is the average stress and $\psi$ is a new variable, as follows [9], [14], [17]

$$
\tau_{r r}=\sigma\left(1-\frac{2}{\sqrt{3}} s \cos 2 \psi\right), \tau_{r \theta}=-\sigma s \sin 2 \psi, \tau_{\theta \theta}=\sigma\left(1+\frac{1}{\sqrt{3}} s \cos 2 \psi\right) .
$$

The above parametrization "solves" the yield condition. The remaining unknowns $(\sigma, \psi)$ are determined by solving the equations of equilibrium (2.1), which now only yield two ordinary differential equations. The corresponding boundary value problem can easily be solved numerically. We omit the details, see, e.g., [5]. A comparison in terms of the average stress $\sigma$ between results from the ODE code using the Sokolovskii variables on the one hand, and results from the present approach on the other hand, is illustrated in Figure 5.1. The agreement is excellent (relative error $<.02 \%$ ).

Having gained some confidence in the approach, we now apply it to the general full problem. Results are presented for the following kind of hoppers. For $0 \leq \lambda \leq 1$, we consider the following family of domains

$$
\mathcal{C}(\phi)=(1-\lambda) \theta_{w}+\lambda \arctan \left(\frac{\tan \theta_{w} \cos \phi_{w}}{\cos \phi}\right)
$$




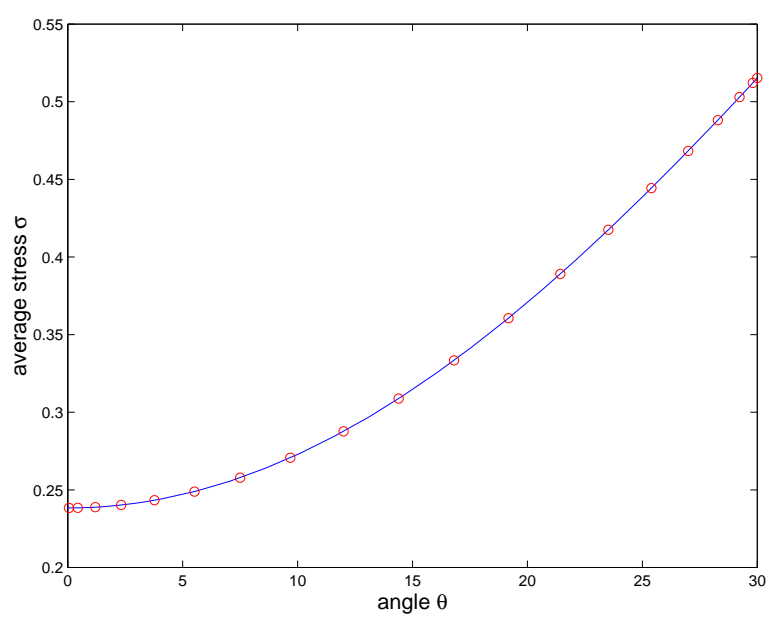

FIG. 5.1. Comparison between ODE solver (solid curve) and present spectral PDE solver (०) for a circular (axisymmetric) cone; $\theta_{w}=30^{\circ}, \delta=30^{\circ}, \mu_{w}=\tan \left(15^{\circ}\right), N=M=20$.

corresponding to

- conical hopper with circular cross-section $\lambda=0$,

- pyramidal hopper with transitional cross-section $0<\lambda<1$,

- pyramidal hopper with square cross-section $\lambda=1$,

In each case treated below, $\phi_{w}=\pi / 4$ and $\theta_{w}$ is a free parameter, both angles being defined in Figure 2.2.

The results presented below are meant to illustrate the qualitative effects of the geometry of the hopper on the flows. All results are represented on horizontal crosssections taken at the same vertical height. This requires appropriate scaling according to the principle outlined in $\S 2$ (stress $=\mathcal{O}(r)$, velocity $=\mathcal{O}\left(r^{-2}\right)$ ). Unlike the stress, the velocity field, which is computed from (2.4), is only defined up to a scalar multiple. This indeterminacy can be solved by imposing an outflow rate for instance. Incidentally, in practice, the flow through outlets at the bottom of the hopper is almost always imposed, through some kind of feeder device for instance. Here, the velocity fields are normalized to have maximum value 1. In Figure 5.2, a comparison between a circular conical hopper, a transitional hopper $\lambda=1 / 2$ and a square pyramidal one with "same" opening angle is offered. By same angle, we mean that at a given common height, the geometric domains corresponding to horizontal cross-sections admit inscribed circles of equal diameter. The material constants are chosen as $\delta=40^{\circ}$ and $\mu_{w}=\tan \left(20^{\circ}\right)$.

All calculations were done on a quarter domain (the solid diagonal line appearing in all figures is an artifact of the plotting software). Several observations can be drawn from the above experiment. First, flows in pyramidal hoppers seem to exhibit larger stresses than flows in purely conical containers. To derive more precise practical conclusions, arguments related to the volume of material effectively contained would have to be considered. Second, and somewhat surprisingly, the presence of corners seems to have relatively little effect on the velocity flow. Comparisons with both laboratory experiments and different plasticity models would be extremely interesting. In the usual axisymmetric case, the latter kind of comparison can be easily performed. Models based on a Tresca yield condition for instance, rather than the present von 

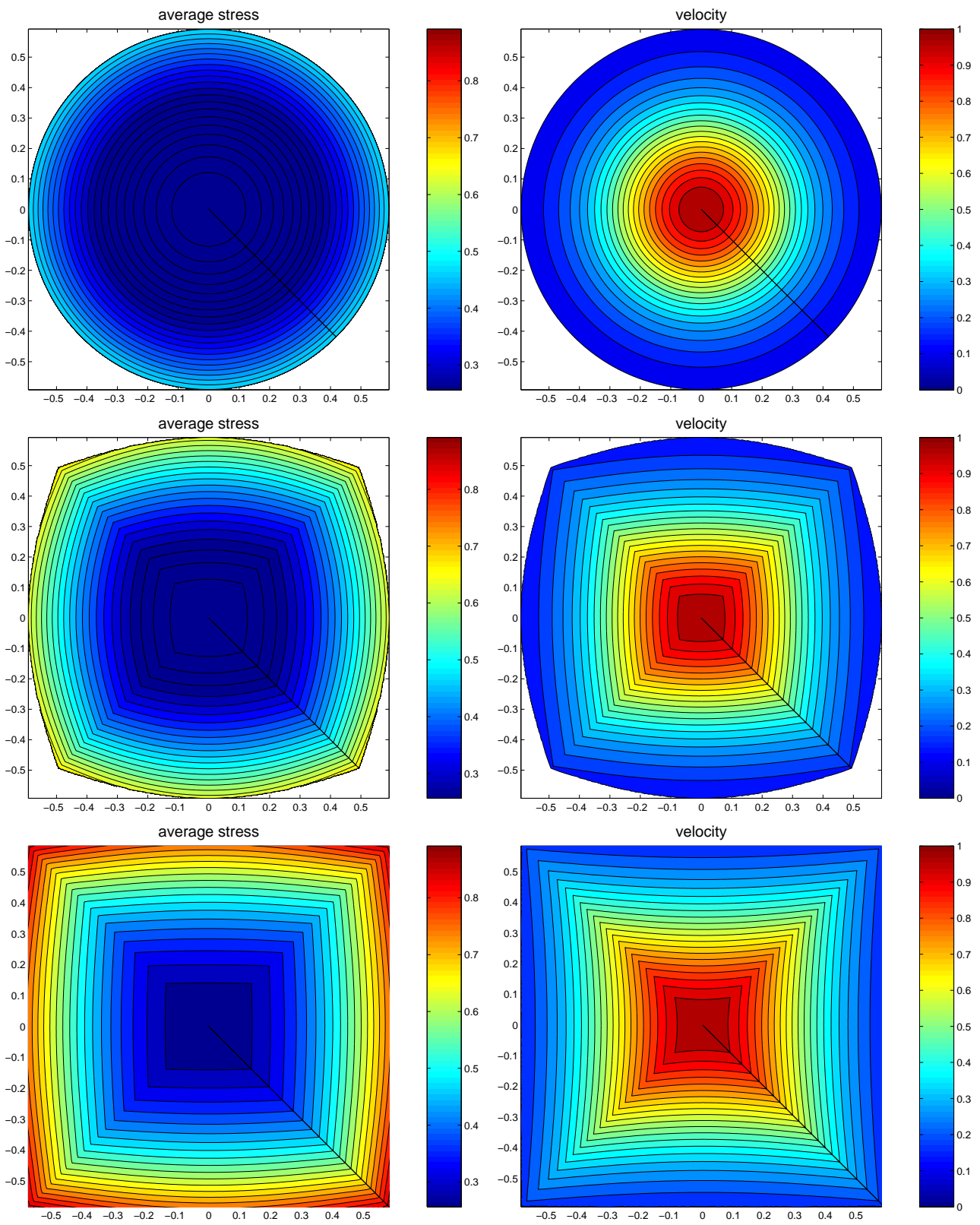

FIG. 5.2. Horizontal cross-sections of flows in similar hoppers (see text) of various shapes; $\theta_{w}^{\text {cone }}=30.68^{\circ}, \theta_{w}^{1 / 2}=35.01^{\circ}, \theta_{w}^{\text {pyramid }}=40^{\circ}, \delta=40^{\circ}, \mu_{w}=\tan \left(20^{\circ}\right), N=M=20$.

Mises condition, tend to predict flows that are more sensitive to wall friction, i.e. the material does not flow as well near the wall, [5], [13], [14]. ${ }^{2}$ Unfortunately, how to generalize Tresca's yield condition to non-axisymmetric flows is not entirely straightforward, as one looses the type of symmetry properties that are precisely used to close

\footnotetext{
${ }^{2}$ It is also interesting to note that in that case, the steady state equations are always hyperbolic. In depth numerical calculations under a Tresca yield condition can be found in [4], [13].
} 
the system in the usual case; see [17], p.29, for further comments.

6. Conclusions. We have shown how to generalize to non-axisymmetric containers the notion of similarity solutions introduced by Jenike [8] in the case of purely conical (or wedge) containers. This generalization comes at the price of having to solve a nonlinear Helmholtz equation on a part of the sphere, as opposed to a boundary problem for a simple system of ODEs in the previous cases.

The present approach applies to "conical" domains, in the mathematical sense, i.e., any domain invariant under the transformation $(r, \theta, \phi) \mapsto(c r, \theta, \phi)$, where $c>$ 0 . It is acknowledged that, clearly, not all industrial hoppers satisfy this property. However, failing this, no similarity solutions are expected to exist, and the full threedimensional equilibrium equations (2.1) would have to be solved.

Acknowledgments. The author thanks Tim Kelley, Matt Matthews, Tony Royal, David Schaeffer and Michael Shearer for many helpful discussions.

\section{REFERENCES}

[1] L. Brillouin, Les lois de l'élasticité en coordonnées quelconques, Congrès International de Mathématique, Toronto, 1924, Annales de Physique, 3 (1925), pp. 251-298.

[2] C. Canuto, M.Y. Hussaini, A. Quarteroni and T.A. Zang, Spectral Methods in Fluid Dynamics, Springer-Verlag, 1988.

[3] I.S. Gradshteyn and I.M. Ryzhik, Table of Integrals, Series and Products, Academic Press, 1965.

[4] P.A. Gremaud and J.V. Matthews, On the Computation of Steady Hopper Flows: I, Stress Determination for Coulomb Materials, NCSU-CRSC Tech Report CRSC-TR99-35, to be published in J. Comput. Phys.

[5] P.A. Gremaud, J.V. Matthews and M. Shearer, Similarity solutions for granular materials in hoppers, in: Nonlinear PDE's, dynamics, and continuum physics, J. Bona, K. Saxton and R. Saxton Eds., pp. 79-95, Contemporary Mathematics, \#255, AMS, 2000.

[6] P. Grisvard, Elliptic Problems in Nonsmooth Domains, Pitman, 1985.

[7] R. JACKSON, Some mathematical and physical aspects of continuum models for the motion of granular materials, in: Theory of Dispersed Multiphase Flow, R.E. Meyer Ed., pp. 291-337, Academic Press, 1983.

[8] A.W. Jenike, Gravity flow of bluk solids, Bulletin No. 108, Utah Eng. Expt. Station, University of Utah, Salt Lake City (1961).

[9] A.W. JenIKe, A theory of flow of particulate solids in converging and diverging channels based on a conical yield function, Powder Tech., 50 (1987), pp. 229-236.

[10] C.T. KelLEY, Iterative methods for linear and nonlinear equations, SIAM, Frontiers in Applied Mathematics \#16, 1995.

[11] T.M. Knowlton, J.W. Carson, G.E. Klinzing and W.C. Yang, The importance of storage, transfer and collection, Chem. Eng. Prog., 90 (1994), pp. 44-54.

[12] P. Lancaster and M. Tismenetsky, The Theory of Matrices, 2nd Ed., Academic Press, 1985.

[13] J.V. Matthews, An analytical and numerical study of granular flows in hoppers, PhD Thesis, Dept. of Mathematics, North Carolina State Univ., 2000.

[14] R.M. Nedderman, Static and kinematic of granular materials, Cambridge University Press, 1992.

[15] E.B. Pitman, The stability of granular flow in converging hoppers, SIAM J. Appl. Math., 48 (1988), pp. 1033-1052.

[16] J.R. Prakash AND K.K. RaO, Steady compressible flow of cohesionless granular materials through a wedge-shaped bunker, J. Fluid Mech., 225 (1991), pp. 21-80.

[17] D.G. SCHAEFFER, Instability in the evolution equations describing incompressible granular flow, J. Diff. Eq., 66 (1987), pp. 19-50.

[18] D. Schulze, Storage, Feeding, Proportioning, in: Powder Technology and Pharmaceutical Processes, Handbook of Powder Technology Vol. 9, D. Chulia, M. Deleuil and Y. Pourcelot Eds, pp. 285-317, Elsevier, 1994.

[19] I.S. Sokolnikoff, Mathematical Theory of Elasticity, McGraw-Hill, 1956. 
[20] A.J.M. Spencer, Remarks on coaxiality in fully developed gravity flows of dry granular materials, in: IUTAM Symposium on Mechanics of Granular and Porous Materials, N.A. Fleck and A.C.F. Cocks Eds, pp. 227-238, Kluwer, 1997. 